\title{
The Advantages and Disadvantages of Machine Translation from the Perspective of Translation
}

\author{
Ying Peng \\ School of Foreign Languages, East China University of Technology, Shanghai, China \\ Email: py@ecust.edu.cn
}

How to cite this paper: Peng, Y. (2021) The Advantages and Disadvantages of Machine Translation from the Perspective of Translation. Open Access Library Journal, 8: e7639.

https://doi.org/10.4236/oalib.1107639

Received: June 11, 2021

Accepted: September 26, 2021

Published: September 29, 2021

Copyright $\odot 2021$ by author(s) and Open Access Library Inc.

This work is licensed under the Creative Commons Attribution International License (CC BY 4.0).

http://creativecommons.org/licenses/by/4.0/

\section{(c) (i) Open Access}

\begin{abstract}
With the rapid popularization of machine translation, there are always differences between machine translation and human translation, and there are also many differences between machine translation softwares. This paper first analyzes the advantages and disadvantages of AI translation through the comparison of different types of human-machine translation examples, and then through the comparison of examples, this paper analyzes and compares two widely used machine translation softwares-Baidu translation and Google Translation, and discusses their advantages and disadvantages. Thus, hopefully this essay can provide reference information for machine translation users and developers.
\end{abstract}

\section{Subject Areas}

Artificial Intelligence, Linguistics

\section{Keywords}

Human Translation, Machine Translation, Functional Comparison

\section{1. 引言}

随着 Internet 的迅速普及, 机器翻译显示了日益广阔的应用前景, 但其 与人工翻译之间的差异始终存在, 且各机器翻译软件之间也存在许多不同之 处。

对于翻译软件使用者和开发者, 本文通过对人机翻译的标准、手段、实 例进行分析，对使用者及开发者，提供借鉴和参考。

对于广大的使用者, 使用何种翻译软常常是随机的, 本文针对两款常用 
机器翻译软件, 通过实例比较, 希望给予不同目的的使用者提供一定的指导。

\section{2. 机器翻译与人工翻译的标准和手段}

\section{1. 机器翻译}

机器翻译是利用计算机把一种自然源语言转变为另一种自然目标语言的 过程。机译系统是随着语料库语言学的兴起而发展起来的, 不同类型的机译 系统由不同的成分构成，但所有机译系统的处理过程都包括以下步骤：对源 语言的分析或理解, 在语言的某一平面进行转换, 按目标语言结构规则生成 目标语言。现在市场上翻译技术相对成熟的是谷歌翻译, 其翻译背后的技术 为基于统计的机器翻译方法, 基本运行原理是通过搜索大量网页内容量的双 语, 将其作为语料库, 然后由计算机自动选取最为常见的词与词的对应关系, 最后给出翻译结果。

机器翻译的过程可以分为原文分析、原文译文转换和译文生成 3 个阶段。 根据不同方案的目的和要求, 既可以将原文译文转换阶段与原文分析阶段结 合在一起，而把译文生成阶段独立起来，建立相关分析独立生成系统，这适 用于研究一种语言对多种语言的翻译。同样也可以把原文分析、原文译文转 换与译文生成分别独立开来, 建立独立分析独立生成系统, 而在研究多种语 言对多种语言的翻译时，宜于采用这样的系统。

\section{2. 人工翻译}

人工翻译主要指通过人工的方式将一种语言转化成另一种语言的行为, 是一种可控制翻译质量的方式。一般两种语言社会的文化习俗存在较大差异, 而这种文化差异影响着翻译活动的范围和方式, 制约着翻译内容的呈现和翻 译策略的选择, 这时候就需要对目标语言的各种形态做充分的了解和研究, 以达到 “信、达、雅”。

人工翻译则是通过人脑中知识的储备和对已有知识的处理分析来达到源 语言与目标语言之间的转换。翻译流程主要包括译、审、校三个环节。最初, 译审工作程序有七个步骤, 即: 翻译、一审、二审、三审、录入、校核、终 审。几年实践后译审室精简了工作流程, 改为翻译、译审、校录、终校、抽 查五道程序 [1]。

\section{3. 机器翻译与人工翻译性能对比}

接下来本文将通过对不同类型人机翻译实例的对比，来分析人工智能翻 译的优缺点。(机器翻译均采用谷歌翻译的译文)

例 1：短句翻译

原句: A free press is essential for peace, justice and human rights for all. 谷歌翻译：新闻自由对所有人的和平、正义和人权至关重要。

人工翻译：新闻自由对于为所有人实现和平、正义和人权至关重要。

例 1 选用相对简短的句型, 没有复杂的句子和语法结构。在此句的翻译 中, 机器翻译和人工翻译类似, 满足了译文准确的要求。 
例 2: 长句翻译

原文: This is what I would like to discuss with you today: first, the early indications of progress under the Belt and Road Initiative-the blossoms-and then, equally important, how to make sure this endeavor can fully deliver in bearing fruit.

谷歌翻译: 这就是我今天要与您讨论的内容: 首先, “一带一路” 倡议 下的进展的早期迹象一一开花一一然后, 同样重要的是, 如何确保这一努力 能够充分发挥作用。

人工翻译: 而这也是我今天想与各位讨论的内容：首先，“一带一路” 的成果已初现端倪, 绽放出了花朵; 其次, 同样重要的是, 如何确保这一努 力结出累累硕果。

例 2 与例 1 相比, 选取了相对复杂的句子。在此句的翻译中, 谷歌翻译 的结果显得不是那么尽如人意。句子结构不够完整, 译文内容不够通顺, 仅 能勉强表达出原文的含义。

例 3: 散文翻译:

原文: Springs are not always the same. In some years, April bursts upon our Virginia hills in one prodigious leap-and all the stage is filled at once, whole choruses of tulips, arabesques of forsythia, cadenzas off lowering plum. The trees grow leaves overnight.

谷歌翻译: 弹簧并不总是一样的。在一些年里, 四月在弗吉尼亚州的山 丘上突然爆发了一次巨大的飞跃一一所有的舞台都立刻被填满, 郁金香的整 个合唱团, 连尧的蔓藤花纹, 降低梅花的华彩。树木一夜之间生长。

人工翻译: 春天并非总是一模一样。四月, 有时不知怎的一跃, 就来到 了弗吉尼亚的山坡上一一转眼到处生机勃勃。郁金香组成了大合唱, 连尧构 成了阿拉伯式图案, 洋李唱出了婉转的歌声。一夜之间, 林木着装, 绿叶瑟 瑟。

例 3 选取了一段散文。在这段散文的翻译中, 我们可以看出机器翻译已 经开始无法胜任翻译的任务了。除了将 “春天” 译为了 “弹簧” , 还有一些 其他的错误。同时, 机器翻译也完全无法达到散文翻译中语言风格优美自然 的要求。

例 4: 诗歌翻译:

原文: It is autumn; not without,

But within me is the cold.

Youth and spring are all about;

It is I that have grown old.

谷歌翻译: 这是秋天; 不是没有, 但在我内心是感冒。

青春和春天都是关于; 我变老了。

人工翻译: 秋天来了; 不是在外界, 是我心里感到了秋凉。

四处都是青春和美景, 只有我已经老迈苍苍。

例 4 是一段诗歌。从结果可以看出, 此时机器翻译几乎处于乱译的状态, 完全无法从中了解到原文的含义, 更别提诗歌的优美性了 [2]。 
例 5: 科技文献翻译

原句: One of the valves in the engine must have gone wrong.

谷歌翻译: 发动机中的阀门之一必须出错。

人工翻译: 发动机的一个气门肯定出了问题。

例 5 是一段科技文献。在汽车制造业, valve 作为汽车零件, 不是通常意 义上的 “阀门” , 而是发动机的 “气门”。机器翻译无法辨识特定应用语境 中词汇多种含义的正确对应, 出现了错误的翻译结果。有时候, 在一些关键 的事物上, 一个小的失误也有可能带来大的损失。机器翻译同时也将 gone wrong 译作了 “出错”, 而不是更符合语言习惯的 “出了问题” 。

原句: The $20^{\text {th }}$ century will not be remembered as the era when space was conquered, or the power of the atom, harnessed, but that in which were made the first machines having intelligence.

谷歌翻译：二十世纪不会被记住为空间被征服的时代，或原子的力量， 被利用，而在第一台有智慧的机器。

人工翻译: 如果在 20 世纪, 人类不制造出具备智能的首批机器, 这一时 代，也不会作为 “征服宇宙的世纪” 或 “原子能利用的世纪” 而被人铭记。

可见, 就科技英语而论, 理解原文的过程, 在多数情况下, 是一个语义 辨认, 语法分析和逻辑分析三方面交互作用的过程, 而不单单是一个逻辑分 析的过程, 请看下面的例句。

例 6: 原句: Shortly before the uninhabited space station reached orbit in May 1973, aerodynamic pressure ripped off a meteoroid and heat shield.

谷歌翻译: 1973 年 5 月无人空间站到达轨道之前不久, 空气动力学压力 剥离了流星体和隔热罩。

人工翻译: 在 1973 年 5 月无人太空站到达轨道前不久, 它的一个防流星 体和防热的护罩被空气动力的压力剥离。

首先, 从逻辑上看, 说: “空气动力压力扯破了一个流星体” , 是不合 事理的, 荒翏可笑的。其次, 从语言上看, 不定冠词 a 是说明 shield 的, 而 不是说明 meteoroid 的。通过逻辑分析和语言分析可以清楚地看出, 名词 meteoroid 和名词 heat 原来都是名词 shield 的定语。A meteoroid and heat shield 的意思是：一个防流星体和防热的护罩。而想要对文字信息实现这种层次的 理解判断, 必须具备人脑的认知与联想 [3]。

例 7: 数字的翻译

原句: Certainly, the kind of measures envisaged by article XIV, the article on domestic regulation, could be kept out of negotiations, at least until the next round started up in 2000.

谷歌翻译：当然，第十四条设想的措施，即关于国内监管的条款，可以 保持在谈判之外, 至少在 2000 年下一轮启动之前。

人工翻译: 当然, 第 16 条所拟订的在本土市场的管理条例, 可留待 2000 年开始的新一轮谈判。

在例 7 中, 包含了数字 “XIV”。从例句中可以看出, 机器翻译得出的 结果尽管后半句存在巨大的误译, 但是在数字的翻译方面上却略胜一筹, 人 
工翻译有时会在数字的处理上存在错误, 但是机器翻译可以完全地避免这一 错误的发生 [1]。

例 8: 惯用语的翻译:

原句: The last thing I want to do is hurt you. But is's still on the list.

谷歌翻译: 我想要做的最后一件事就是伤害你。但仍然在名单上。

人工翻译：我真不想伤害你, 但你也别逼我。

在例 8 中, 机器翻译处理的不妥, 表达出了与原文相反的含义。

例 9: 网络用语:

原句: 卡死了。

谷歌译文: The card is dead.

人工翻译: The net is busy. (网速慢); I've got stuck (游戏画面滞后); The game frames are blocked (frame 指游戏的帧)。

在例 9 中, 机器无法考虑语境意义, 只按照字面句法结构, 把 “卡” 作 为主语, “死了” 作为谓语。无法根据实际情况作出判断, 选择合适的表达 方式[4]。

从以上 9 个例子, 我们可以得出结论: 人工智能在进行非文学翻译, 翻 译较简单句子时, 能基本良好地完成句子的翻译, 而对于长难句的翻译处理 能力仍需大幅提升。而当在进行文学翻译时, 如散文、诗歌等, 人工智能不 仅不能像人工的译者实现 “信达雅” 的全部要求, 就连最基本的 “信” 都很 难达到。在进行一些惯用语和生活用语翻译的时候, 人工智能也无法结合具 体体况做出理解和选择, 导致意义表达的不准确甚至是错误。

相较于机器翻译, 目前人工翻译仍占据着较大的市场。在许多正式场合, 大一些的比如国际、国家级会议，小一些的如到公司间的谈判，人工翻译仍 是无法被替代的。究其根本原因, 人工翻译的准确率仍然大于机器翻译, 专 业的人工翻译更是如此。但是不可否认的是, 机器翻译的发展仍然保持着高 速的发展, 现阶段的机器翻译最大的优势莫过于其超快的翻译速度。人工翻 译和机器翻译作为现今最主要的两种翻译方式, 二者在翻译方式、结果、准 度及应用领域四个方面存在差异。机器翻译方式虽在整体翻译准度面不及人 工翻译，但其速度快的巨大优势满足了现今对翻译工作越来越大的需求量。 而人工翻译虽不及机器翻译来的效率高, 却是对语言本身一次次的精雕细玩, 其中凝练着译者的心血，反映着不同的文化。尽管机器翻译和人工翻译之间 存在着一定的竞争关系, 但两者应取长补短, 共同促进发展 [1] [2]。

\section{4. 常用机器翻译性能对比}

反映迅速发展的背景下，翻译软件作为帮助人们消除 “语障” 的工具， 被越来越多的人认识和使用, 目前国内市场上有许多翻译软件, 如百度翻译、 谷歌翻译、有道翻译、灵格斯翻译、Babylon 和金山词霸等。本文将通过对两 款国内款常用机器翻译软件的分析对比, 帮助广大用户更好地按需选择。

\section{1. 百度翻译}

百度翻译支持 28 种热门语言之间的互译。百度的优势在神经网络技术的 
运用上。相对而言, 传统的统计翻译方法是基于短语或者句法翻译的, 只能 达到局部解码的效果; 神经网络翻译的突破是先理解整个句子的意思, 然后 再翻译, 使得其所选翻译词语更符合句子的语意。但神经网络翻译需要大量 的双语语料, 以便进行数据训练, 获得优异的效果。中英互译时可以获得很 多现有双语语料，但在中文到日文、韩语、泰语等翻译上很少有大规模的语 料。所以中译英、英译中的神经网络翻译占到百度翻译的 $90 \%$ 以上, 而小语 种的语料没有那么多, 在翻译时还是多数使用统计翻译。值得注意的是, 神 经网络翻译目前还只是处于高级一点的模式识别技术阶段, 没有真正解决语 义问题, 语义理解是机器翻译领域接下来要解决的重要问题。

\section{2. 谷歌翻译}

谷歌翻译软件提供 102 种语种之间的互译。谷歌采用的就是统计机器翻 译的方法, 通过对大量平行语料进行统计分析, 构建统计翻译模型, 进而使 用此模型进行翻译。简单来说, 谷歌翻译在生成译文时, 会在大量人工翻译 的文档中查找各种模型, 进行合理的猜测, 从而得到恰当的翻译。由于机器 操作, 谷歌翻译对某种特定语言可供分析的人工翻译文档越多, 译文的质量 就越高。谷歌翻译通常会提供备选翻译，当用户点击它时，谷歌会记下反馈。 此外, 在翻译完成之后, 如果用户觉得效果不佳, 自己有能力修正其中的错 误或换种更通顺的译文形式，则可向谷歌提供 “更好的翻译建议”，以帮助 其提高翻译质量。通过这种 “众包” 的方式, 谷歌翻译不断改进该语境的翻 译质量。

\section{3. 百度翻译、谷歌翻译对比}

翻译的本质是意义的双语转换, 即把一种语言所承载的信息转移到另一 种语言中。转换的主要性能指标是它的有效性, 有效性是指目的语在编码过 程中要保留与原文同样多的信息量和保留原文的信息重心。“信息翻译强调 的是信息和意义的传递, 它要求打破语言的外壳取出其实质。因此, 句法作 为语言的外壳应彻底抛开。信息与语言此时就像商品与包装的关系。”所以, 合格的译文必须忠实于原文的内容, 实现译文信息的完整性。

为了更全面的评估这两个在线翻译的翻译水平, 将对谷歌翻译和百度翻 译分别进行不同语言单位、不同语言方向和不同文本类型的翻译测评。在不 同语言单位层面，分别对专有名词、短语和段落进行测试; 在不同语言方向 上，分别进行了汉英和英汉测试; 在不同文本类型中，分别选取了专业性文 本、文学类文本、日常用语类文本进行测试。

下面，用几个具体例子来对比百度与谷歌文本翻译情况。

例 1：专有名词翻译
A. 地理名词
原文: Loess Plateau
谷歌翻译：黄土高原
百度翻译：黄土高原
B. 机构名称
原文: Chinese Academy of Social Sciences 
谷歌翻译: 中国社会科学院

C. 历史名胜

原文: the Forbidden City

谷歌翻译: 故宫

D. 小说

原文: A Tale of Two Cities

谷歌翻译: 双城记
百度翻译：中国社会科学院

百度翻译：紫禁城

除了最后一个专有名词百度没有将查尔斯 - 狄更斯的长篇小说的书名翻 译出来, 两种工具的翻译结果基本一致, 并且准确度高。其一说明机器翻译 在专有名词的翻译上比较在行; 其二, 对专有名词的翻译可能谷歌翻译在有 些情况下略胜一筹, 但大部分情况依然和百度翻译棋逢对手。

例 2: 短语翻译

A. 原文: up to snuff

谷歌翻译: 到鼻烟

百度翻译：到达标准

B. 原文: squeaky clean

谷歌翻译: 非常干净

百度翻译：非常干净的、道德高尚的

C. 原文: bounce back

谷歌翻译: 反弹百度翻译: 卷土重来

D. 原文: weigh in

谷歌翻译: 称量体重

百度翻译: 发表评论、施加影响

上述例子来看, 谷歌的翻译停留在对单个单词的理解上, 百度翻译有时 不仅能译出其直接意思, 还能译出短语的深层含义。笔者还选取了其他一些 短语测试, 但是测试结果总体显示百度翻译对于短语的翻译更加准确, 译文 的忠实度和可懂度当两个单词组成一个与其原来各自意义相差较大的词组 时, 谷歌并不能翻译出其生成的新意, 而百度在这点上比较灵活高于谷歌翻 译。除此之外, 百度翻译在短语翻译方面, 还添加了谷歌所不具备的 “示例 用法” 的板块, 这对于英语学习者非常实用。

例 3: 日常用语翻译。

A. 原文: 我已经听够了。

谷歌翻译: I've heard enough. 百度翻译: I've heard enough.

B. 原文：你要我怎么说你才能明白呢?

谷歌翻译: What do you want me to say?

百度翻译: What do you want me to say?

C. 原文: You really flatter me.

谷歌翻译：你真的爱我。

百度翻译: 你过奖了。

通过测试发现, 谷歌翻译和百度翻译都能将日常用语翻译好, 并且两者 翻译水平相当[5]。

例 4: 中译英长句翻译

原文：法国数据保护机构 “国家信息与自由委员会” 今日宣布，将对 Facebook 处以 15 万欧元(约合 16.6 万元美元)的罚款。

谷歌翻译: French data protection agency "National Information and 
Freedom Committee" announced today that it will be on Facebook to 150,000 euros (about 16.6 million) fine.

百度翻译: The French data protection agency "national information and Freedom Committee" announced today, will be fined 150 thousand euros for Facebook (\$166 thousand) fine.

从上述例子可以看出：原文用中文习惯对两句话进行了断句：“...... 日宣布, 将对……”。谷歌翻译在这里把后半句变成了从句用了一个 That 引 导，从而将第一句改成了更符合英文表达习惯的长句。而百度翻译在这里还 是按照中文的断句, 对英文进行了同样的断句, 属于中式翻译, 不符合英文 翻译的逻辑规律。因此, 谷歌翻译相对于百度翻译来说, 翻译出的文本更地 道自然。

例 5: 中译英短句翻译

A. 原文: 老师喜欢花儿。

谷歌翻译：Teachers like flowers. 百度翻译: The teacher likes flowers.

两个翻译软件在翻译时除了动词的单复数不同, 翻译没有什么区别。

B. 原文：洪荒之力(网络热词)

谷歌翻译: Prehistoric force. 百度翻译: I have give my full play.

洪荒之力是指用尽了全力, 可见百度翻译比较贴切, 而谷歌仅仅是把词 语直译出来, 并没有表达出 “洪荒之力” 的原意。实际上, 不仅 “洪荒之力”, 绝大多数流行网络用语, 谷歌都无法正确识别翻译, 很显然谷歌翻译在短句 翻译时的本土化水平不及百度翻译。

通过上述对比, 可见百度翻译在短句中译英上处理比谷歌准确, 而谷歌 则在长句的中译英处理上比百度准确。

例 6: 英译中文本翻译

原文: “You have weighed the pros and cons and carefully assessed the impact. After indepth consideration, you have decided to accept that new job, or launch your own business, or take time off to be with your children. You know it's the right choice-but your boss, friends, and colleagues aren't convinced. What should you do when people you respect disagree with your decisions?”

谷歌的英译中结果比百度出现了更多问题。

比如原句中“or take time off to be with your children”。这里的最佳翻译应 是：或者抽出时间陪陪孩子。

谷歌翻译：或者与您的孩子一起休息。百度翻译：或者抽出时间和你的 孩子在一起。

再比如原句中: “What should you do when people you respect disagree with your decisions?” 这里应该译作：当你尊重的人不同意你的决定时, 你该 怎么做?

谷歌翻译：当你尊重不同意你的决定时，你应该怎么办？

百度翻译：当你尊重的人不同意你的决定时，你应该怎么做?

可以看到英译中谷歌翻译还是不够准确的, 经常会遇到句子不通顺, 结 构不对的问题。相比之下, 百度翻译的翻译结果更加注重译文的规范和流畅。 
软件翻译需要借用加减词法、拆句与合句法等手段, 同时也需借助词性、语 态、时态及肯定与否定句等句法结构的转换技巧。百度翻译在运用上述翻译 技巧上, 在词性转换和词序调换方面完成的比谷歌翻译好, 在增词和减词方 面两者都表现一般，而在融合简单句、拆分复合句的技巧方面都表现较差[6]。

\section{4. 机器翻译的使用技巧总结}

经过上述对比, 可见百度翻译和谷歌翻译各有所长。百度翻译的单词丰 富度、扩展度高并且中译英短句翻译及英译中文本翻译质量高; 而谷歌翻译 中译英长句翻译更为准确。因此在进行不同语言单位的文本翻译时, 短语和 段落的翻译应该选择百度，而专有名词的翻译既可选择百度也可选择谷歌; 在进行不同语言方向的文本翻译时，对汉译英追求地道和流利表达的译文的 译者选择谷歌, 要求语法严谨准确的译者选择百度, 而英译汉时选择百度是 明智之举; 在进行不同类型的文本翻译时, 专业性强的文本选择谷歌翻译, 对日常用语类文本进行翻译的译者两种翻译机器都可以使用, 但切忌使用它 们进行文学翻译。

\section{5. 结论}

我们可以得出结论：1) 机器翻译适合于进行非文学翻译; 人工翻译适用 于文学翻译以及专业领域谈判和会议。2) 百度翻译适用于短语和段落的翻译 和英译汉翻译; 谷歌翻译适用于长句翻译及专业性强的文本和汉译英翻译。

\section{Conflicts of Interest}

The author declares no conflicts of interest.

\section{References}

[1] 訤䒯. 机器翻译与人工翻译的差异一一 《世界贸易组织的新协定》为例 [J]. 中 外交流, 2018(25): 31.

[2] 田江波. 从翻译的标准来探讨机器翻译能否取代人工翻 [J]. 海外英语(上), 2018(7): 126-127.

[3] 罗华珍, 潘正芹, 易永忠. 人工智能翻译的发展现状与前景分析 [J]. 电子世界 2017(21): 21-23.

[4] 潘玥, 朱风兵. 功能对等理论下的人机翻译对比研究[J]. 延安职业技术学院学报, 2015, 29(5): 65-66. https://doi.org/10.3969/j.issn.1674-6198.2015.05.030

[5] 李娴. 谷歌翻译与百度翻译比较研究[J]. 广东经济, 2017(7): 278.

[6] 涂正正. 谷歌翻译与百度翻译 APP 的功能 三维度之比较 [J]. 江西广播电视大学 学报, 2018, 20(1): 72-77. https://doi.org/10.13844/j.cnki.jxddxb.2018.01.014 


\section{Appendix (Abstract and Keywords in Chinese)}

\section{翻译视角下的机器翻译利弊}

摘要: 机器翻译为越来越多的人使用, 但其与人工翻译之间的差异始终存在, 且各机器翻译软件之间也存在许多不同之处。本文首先通过不同类型人机翻 译实例的对比, 来分析人工智能翻译的优缺点, 再通过实例比较, 对目前使 用较广的两款机器翻译软件一一百度翻译和谷歌翻译进行分析对比, 探讨其 优势与不足。为翻译软件使用者和开发者, 提供参考和借鉴。

关键词: 人工翻译, 机器翻译, 功能比较 